\title{
REAL-TIME OPTICAL SPECTRUM ANALYZER
}

MIGUEL A, MURIEL

JOSE A, MARTIN-PEREDA

DEPARTAMENTO dE ELECTRONICA CUANTICA

E.T.S.I, TELECOMUNICACION

UNIVERSIDAD POLITECNICA DE MADRID

CIUDAD UNIVERSITARIA

MADRID - 3 SPAIN

\begin{abstract}
The aim of this paper is to present a real-time optical spectrum analyzer based on diffraction properties of area modulated signals. From the oscilloscope image of a signal we extract its spectra, which presented in another scope.
\end{abstract}




\section{INTRODUCTION}

We present an optical set-up that performs the Fourier transform of a signal represented on a scope. The basis of the system is the fourier properties of area modulation by means of cofierent optical radiation (1aser [. The optical input signal is the oscilloscope image of the studied signal.

OPTICAL TRANSFORMATION OF AREA-MODULATED SIGNAL

Area modulation is a simple operation which produces a binary transmittance of two discrete tones. The basic forms of area modulation, unilateral and bilateral, are described in Fig. 1 . These two forms are chosen in our present. application because of their simplicity. We note that other forms of area modulation may also be used.since the area-modulated signal is in binary form (i.e.,transparent and opaquel.

One can describe the 2-D amplitude transmittance function for a bilateral area modulated signal by

$$
T_{B}(x, y)=\operatorname{rect} \frac{x}{L} \operatorname{rect} \frac{y}{2[B+f(x)]}
$$

and a unilateral area modulated signal by

$$
T_{u}(x, y)=\operatorname{rect} \frac{x}{L} \operatorname{rect} \frac{y-[f(x)-B] / 2}{B+f(x)}
$$

where $(x, y)$ is the spatial coordinate system, $L$ is the length of the input in the $x$ direction, $B$ is the spatial bias level, $f(x)$ is the input signal function, and

$$
\text { rect } \frac{A}{C}= \begin{cases}1, & A \leqslant C / 2 \\ 0, & \text { otherwise }\end{cases}
$$



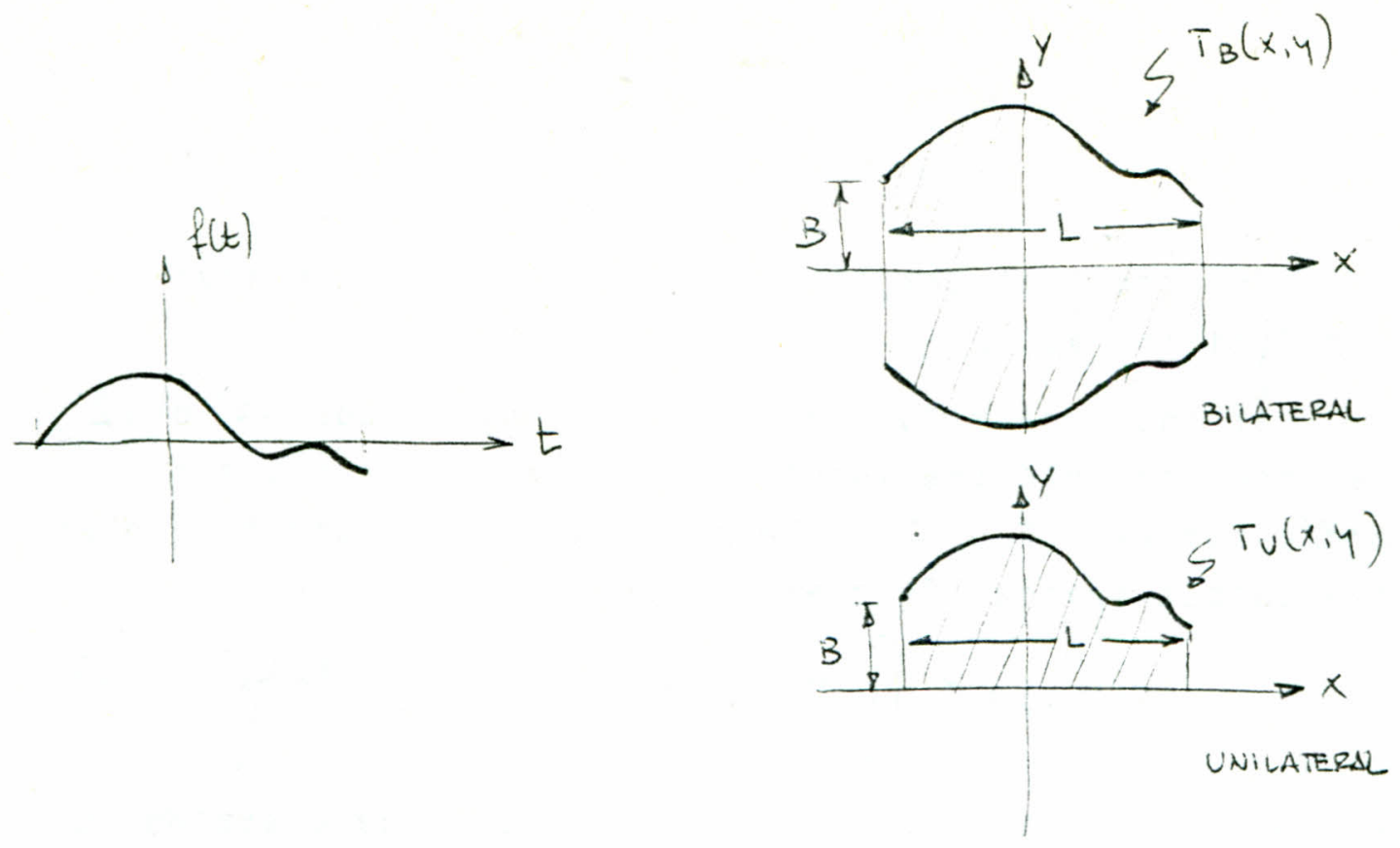

FIG. 1

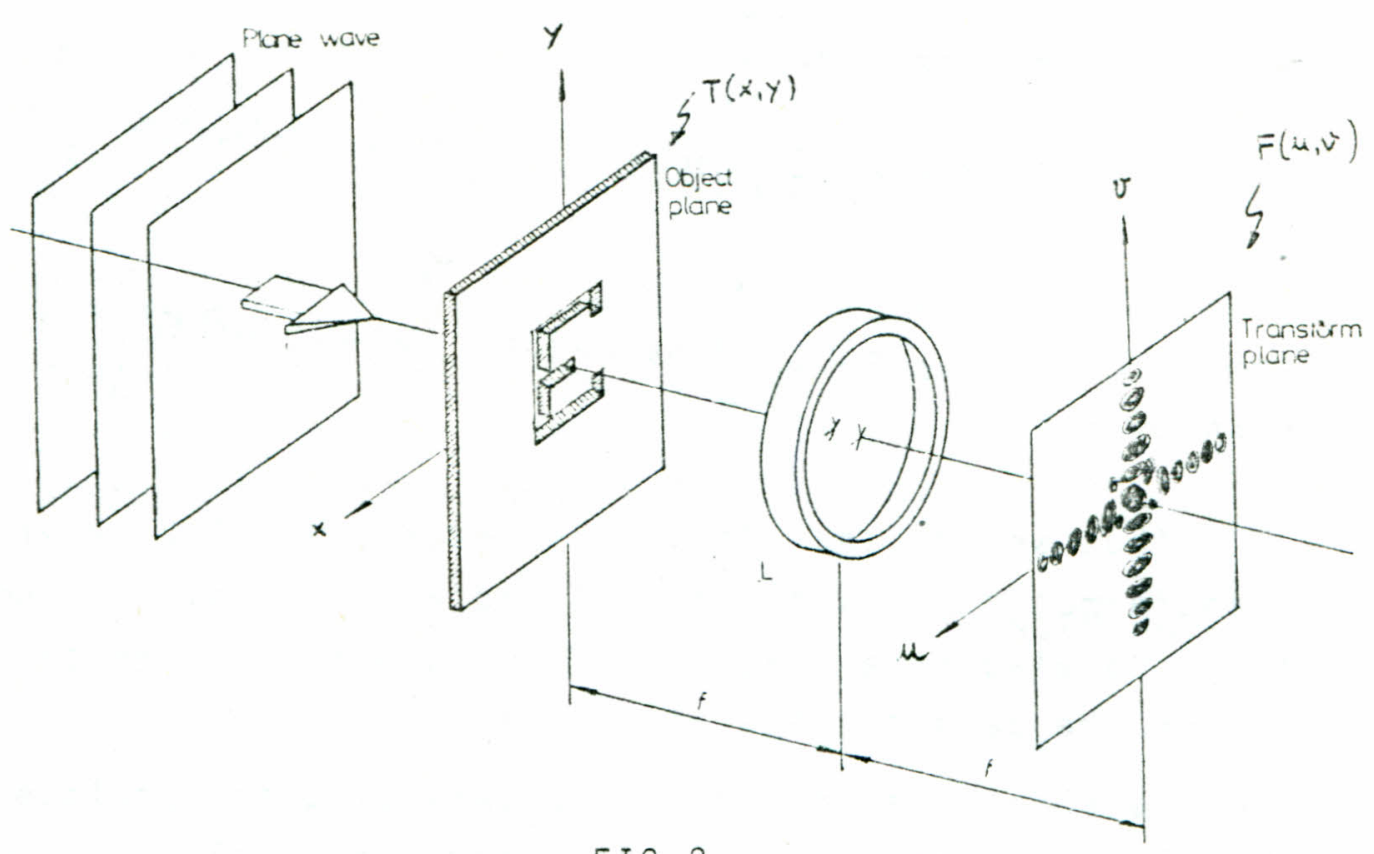

$F I G .2$ 
We note that the bias level is chosen such that $B \geq f(x)$ for a $11 x$.

If the area-modulated transparency of Eqs. (1) or (2) is inserted into the input plane of a coherent optical processor (see Fig.2) the complex light distribution at the output spatial frequency plane will be

$$
F(u, v)=c \int_{-\infty}^{+\infty} \int_{-\infty} T(x, y) \exp [-i(u x+v y)] d x d y
$$

where (u,v) is the spatial frequency coodinate system, C is an arbitrary complex constant, and $T(x, y)$ is the input. We note that Eq.(3) is generally difficult to evaluate. However, if we restricted our evaluation to only the $\mu$-axis spatial frequency coordinate, for the case of the bilateral area modulated signal we have

$$
F_{B}(u, 0)=C \int_{-\infty}^{+\infty} \int_{B}(x, y) \exp [-i u x] d x d y
$$

which can be shown to be

$$
\begin{aligned}
F_{B}(\mu, 0) & =2 C \int_{-\infty}^{+\infty} \operatorname{rect}\left(\frac{x}{L}\right)[B+f(x)] \exp (-i \mu x) d x, \\
& =2 C\left[\int_{-4 / 2}^{4 / 2} B \exp [-i v x] d x+\int_{-4 / 2}^{L / 2} f(x) \exp [-i u x] d x\right]
\end{aligned}
$$

Thus we see that Eq.(5) represents a 1-D Fourier transformation of a constant $B$ and an input signal function $f(x)$. We also note that Eq. (5) is essentially the same form as for the density-modulated case.

We also note that for a unilateral area-modulated signal, the complex amplitude distribution along the $u$-axis coordinate would be 


$$
\begin{aligned}
F_{u}(\mu, 0) & =\iint_{-\infty}^{+\infty} T_{u}(x, y) \exp [-i \mu x] d x \\
& =C\left[\int_{-L / 2}^{L / 2} B \exp [-i \mu x] d x+\int_{-L / 2}^{L / 2} f(x) \exp [-i v x] d x\right] \\
& =\frac{1}{2} F_{B}(\mu, 0)
\end{aligned}
$$

which is identical with the result obtained for the bilateral case, except that the irradiance is reduced by a factor of four.

In analysis, we consider a bilateral sinusoidal areamodulated signal inserted in the input plane of a coherent optical processor, i.e.,

$$
f(x)=A \sin \left(\mu_{0} x+\theta\right)
$$

where $u_{0}$ is some arbitrary angular spatial frequency, and $\theta$ is a constant phase factor. By substitution, we show that

$$
F_{B}(\mu, v)=k_{1} \delta(\mu)+k_{2} \delta\left(\mu-\mu_{0}\right)+k_{3} \delta\left(\mu+u_{0}\right)
$$

where $K$ is a proportional constant. Two spectra points are located at $u=-u_{0}$ and $u=u_{0}$. We see an example of spectrum analysis based on area modulation. In conclusion we have the Fourier transform in the u-axis (Fig.3 ).

\section{INCOHERENT TO COHERENT OPTICAL RADIATION TRANSFORMATION}

The previous analysis is based on the use of coherent optical radiation. So we need to convert the incoherent scope image to coherent optical signal. We get this transfor mation by means of a Hughes Liquid Crystal light valve (Fig.

4 l, how it works?. 


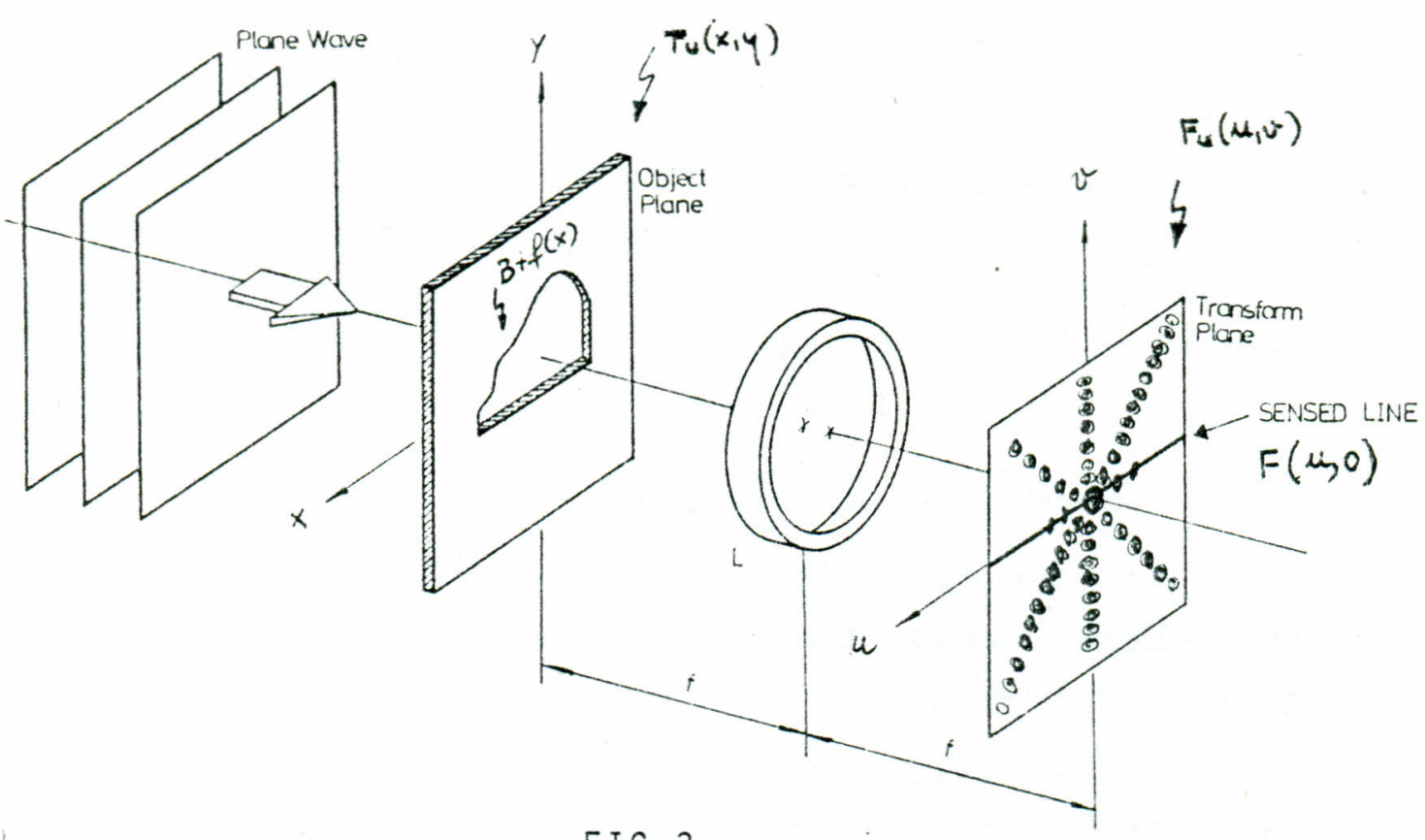

FIG. 3

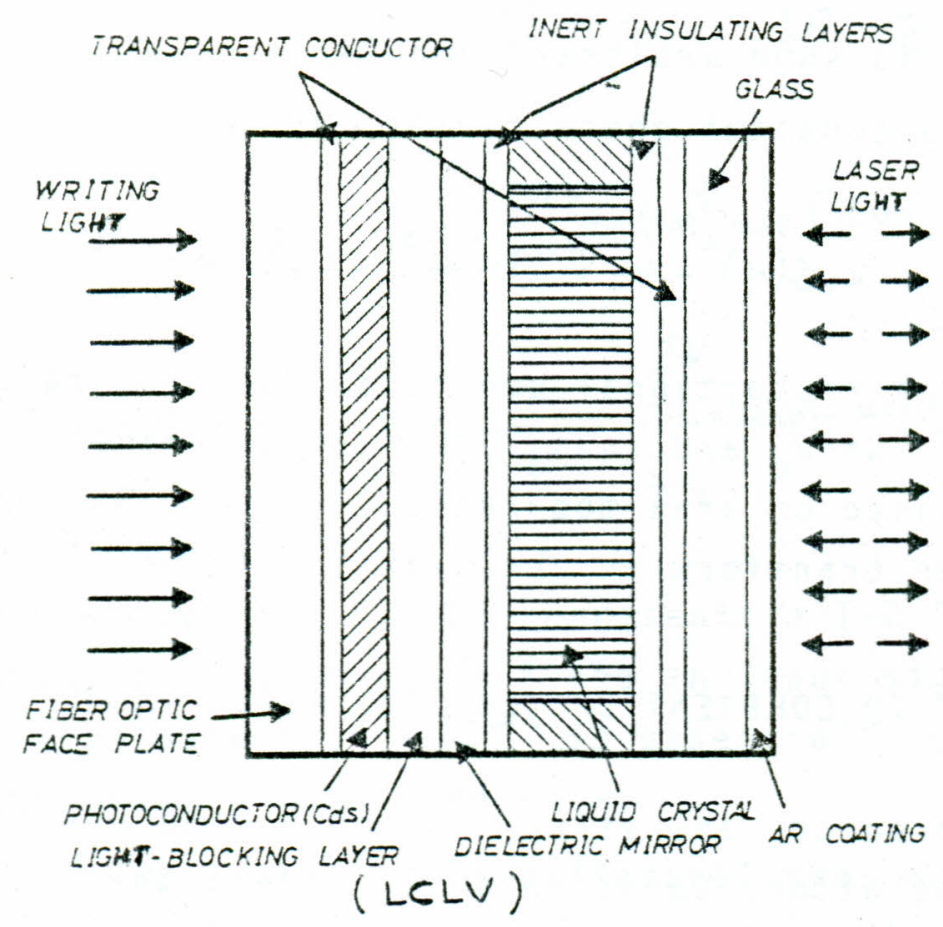

FIG. 4 


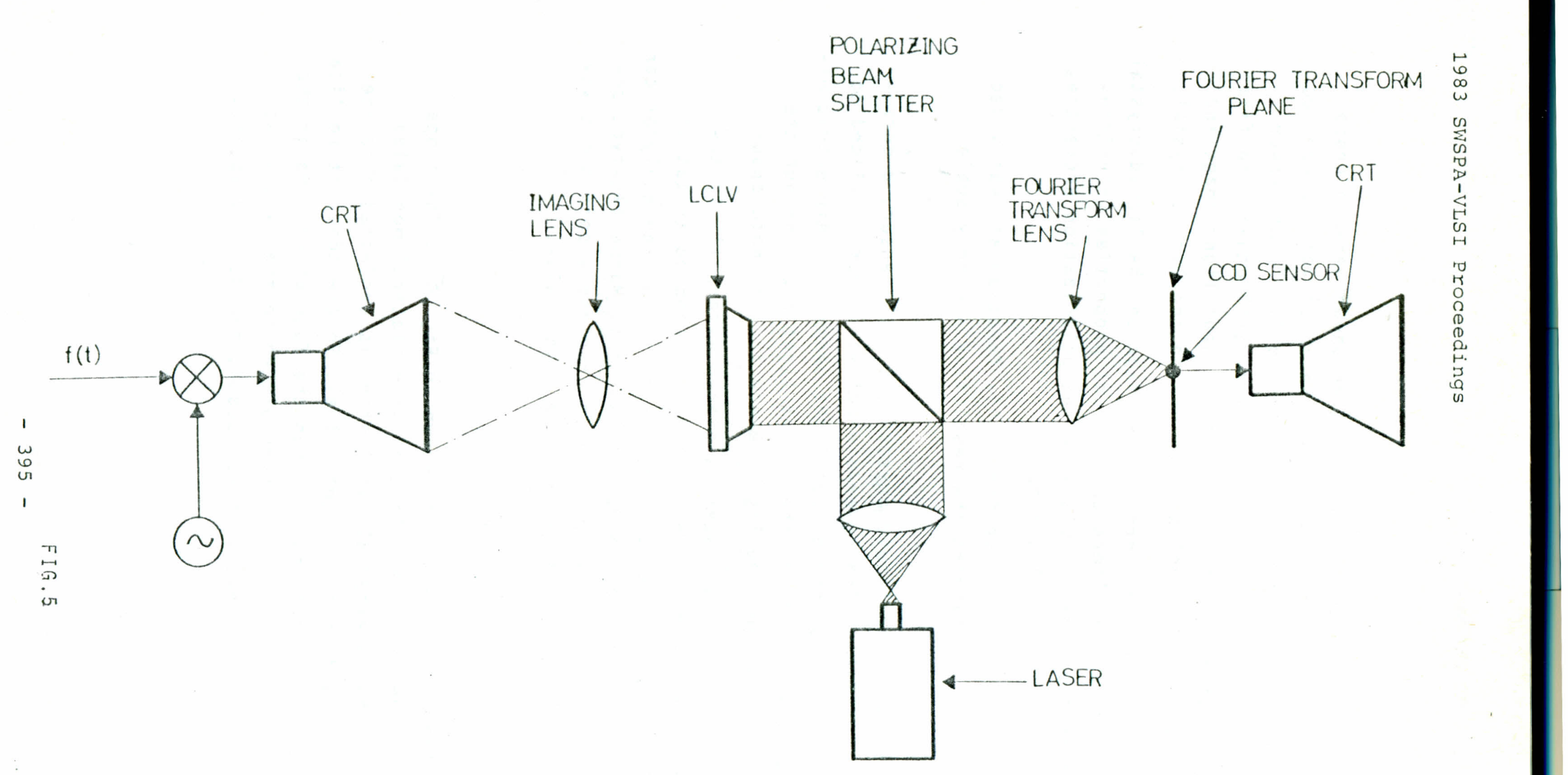


The liquid crystal light valve shown here in cross section is a thin film sandwich with an ac voltage applied across it. It consists of a cadmium sulfide photosensor layer, a liquid crystal layer, and several other thin films. The photosensor detects a low level input light image and impresses a replica voltage pattern on the liquid crystal.

The voltage pattern of the image on the liquid crystal then electrically alters the optical properties (birefringencel of the layer to modulate the polarization properties of the output laser light beam.

The light valve performs this function at near video rates while maintaining the resolution and gray scale quality of the image.

Between the liquid crystal and photosensor, a combination light-blocking layer/dielectric mirror is.placed to

- isolate the input and output light beams. The dielectric mirror serves to reflect the output light back through the liquid crystal and the light lelkage to the photosensor.

Finally, an analyzer is placed externally in the output laser beam to convert the modulated polarization state into a visible multigray level image. The analyzer can be a high extinction ratio nonabsorptive MacNeille-type polarizing beam splitter which also provides a convenient way of directing ouput light into the light valve.

\section{OPTICAL SPECTRUM ANALYZER SET-UP}

The optical set-up is shown in Fig. 5. The scope image converted on a bilateral or unilateral modulated signal with an external oscillator, is projected onto the LCLV (liquid crystal light valveI. So the coherent replica of the input scope image, gives the fourier spectra in the u-axis. With a CCD line sensor placed in the u-axis, we extract and present in other scope, the desired spectrum signal. 